\title{
Hour Times Mole per Liter per Kilogram per Meter Squared
}

National Cancer Institute

\section{Source}

National Cancer Institute. Hour Times Mole per Liter per Kilogram per Meter Squared.

NCl Thesaurus. Code C111222.

Hours times moles per liter, divided by kilograms per meter squared. 